JPE 11-5-8

\title{
Simple Space Vector PWM Scheme for 3-level NPC Inverters Including the Overmodulation Region
}

\author{
Dong-Myung Lee*, Jin-Woo Jung ${ }^{\dagger}$, and Sang-Shin Kwak** \\ ${ }^{*}$ School of Electronic and Electrical Engineering, Hongik University, Seoul, Korea \\ $\dagger$ Division of Electronics and Electrical Engineering, Dongguk University, Seoul, Korea \\ ** School of Electrical and Electronics Engineering, Chungang University, Seoul, Korea
}

\begin{abstract}
This paper proposes a simple space vector PWM (SVPWM) scheme including overmodulation operation for 3-level NPC (Neutral Point Clamped) Inverters. The proposed scheme features a simple decision and calculation procedure for determining switching times in the overmodulation range by utilizing the duty calculation method used in 2-level inverters and the minimum phase error projection method widely employed in motor drive systems. The proposed scheme does not need to detect the angle of the reference vector or calculate trigonometric functions to determine the magnitude of the voltage vector. The magnitude of the angle of the new reference voltage vector is decided in advance with the help of the Fourier Series Expansion to extend the linearity of the output voltage of 3-level inverters in the overmodulation region. Experimental results demonstrate the validity of the proposed SVPWM scheme including overmodulation operation for 3-level NPC inverters.
\end{abstract}

Key Words: 3-level NPC (Neutral Point Clamped) inverters, Overmodulation, Space Vector PWM (SVPWM)

\section{INTRODUCTION}

Inverters are widely adopted in various industrial areas such as variable speed drive systems, and power conditioning system for renewable energy because of their ability to control the magnitude and frequency of output voltage. Recently, to reduce the harmonics of inverter output voltage and to meet the voltage ratings of the power devices used in inverters, multi-level structures like the 3-level inverter scheme have been widely researched, and the adaptation of multi-level, especially 3-level, converters has gradually increased thanks to their advantage of less harmonics [1]. There exists various configurations of multi-level inverters such as neutral point clamped (NPC), flying-capacitor, cascaded H-bridge, etc. [2]. Among these configurations, the proposed SVPWM scheme is devised for NPC inverters, which are the most common configuration of 3-level inverters, and they have the neutral point of the DC link tied to each inverter arm by clamping diodes [3].

The SVPWM method is commonly used in 3-level as well as 2-level systems. There even exist different switching time calculation methods, usually in the linear region. The main idea behind determining the switching time for two and multilevel systems is identical. In other words, the switching time

\footnotetext{
Manuscript received Dec. 2, 2010; revised Jul. 25, 2011

Recommended for publication by Associate Editor Yong-Sug Suh.

$\dagger$ Corresponding Author: jinwjung@dongguk.edu

Tel: +82-2-2260-3348, Dongguk University

${ }^{*}$ School of Electronic and Electrical Engineering, Hongik University, Korea

** School of Electrical and Electronics Engineering, Chungang University, Korea
}

is determined by the equalization of the reference voltage during the switching period with the averaged voltage vector generated by the time assignment of the three different voltage vectors that exist in the vertices of the triangle where the reference voltage exists. On the other hand, there exist several types of SVPWM schemes for 3-level NPC inverters with overmodulation operation [4]-[6]. [4] and [5] suggested overmodulation schemes, which increase the linearity of the inverter output voltages. However, these schemes use trigonometric functions in calculating the modified voltage vector and the switching time itself, and consequently they are relatively complex. The authors of [6] proposed an overmodulation method with a neutral voltage balancing algorithm, which does not use the medium voltage vectors that affect the potential of the DC link voltage level, based on the overmodulation scheme for 2-level inverters suggested in [7].

The overmodulation method can be classified as a so-called static or dynamic one. The dynamic overmodulation method (hereinafter DOM) is used in vector control systems for motor drives. In this scheme, when the reference voltage exists outside of the hexagon, the reference voltage is projected on side of the hexagon. One of the common projection schemes is the minimum phase error method [8]. On the other hand, the so-called static overmodulation method (hereinafter SOM) is employed in $V / F$ control of induction motors to increase the inverter output voltage in the high speed region. In the SOM, according to the modulation index represented by $m$, which varies from zero to one, the inverters generate an output voltage from zero to one having a square waveform 
[9], [10]. In order to apply multilevel inverters in motor drive applications that include high speed operation, it is necessary to develop a scheme for overmodulation.

The proposed scheme obtains the magnitude of a new reference voltage directly from the modulation index and eliminates the decision procedure for the angle and magnitude of the reference vector. On the other hand, in [4] and [5] several steps are involved to determine the reference voltage vectors. Furthermore, the proposed method utilizes the minimum phase error projection scheme for generating the duty ratios. As a result, it has a simple calculation procedure.

Similar to [5], the magnitude of the reference voltage and that of the phase angle are predetermined by the result of the Fourier Series Expansion (FSE) in the proposed method. In implementation, these values are obtained from approximate equations of the magnitude and phase angle corresponding to the modulation index. The experimental results for a 3level NPC inverter in both the linear and overmodulation regions with $\mathrm{V} / \mathrm{F}$ control of an induction motor demonstrate the validity of the proposed SVPWM scheme.

\section{SVPWM FOR 3-LEVEL SYSTEM}

Before explaining the proposed SVPWM scheme including overmodulation, the method for determining the switching time for a 3-level inverter is described first. Among the various ways of selecting the switching time for a 3-level inverter, the proposed scheme uses the scheme for 2-level inverters in order to utilize the overmodulation scheme of 2-level inverters in a 3-level system.

\section{A. Space vector PWM for 3-level inverters}

The 3-level NPC inverter [3] shown in Fig. 1 has 3 different switching states for the pole voltage denoted as 1,0 , and -1 . Where, 1 represents the switching status in which the top two switches (i.e. the first and second switches from the top in an inverter leg) are on, and the resultant pole voltage is $1 / 2 V_{d c}$. The 0 status means that the second and third switches are on while generating a $0 \mathrm{~V}$ pole voltage. The third and fourth switches in one leg are turned on in the case of -1 resulting in a $-1 / 2 V_{d c}$.

Fig. 2 shows a hexagon of the voltage vectors for 3-level inverters. Each inverter leg can have three switching states, which results in 27 voltage vectors. No power is delivered to the load for the switching states (111), (000), and (-1-1-1), which is referred to as the zero voltage vector $(\mathrm{ZV})$. There exists the same voltage level for different switching states, such as (110) and (00-1), having the identical line to line voltages. Hence, the 27 possible voltage vectors are classified as 18 effective voltage vectors and 1 zero vector. The non-zero voltage vectors can have three phase voltage levels, which are $2 / 3 V_{d c}, V_{d c} / \sqrt{3}$, and $V_{d c} / 3$. Each voltage level corresponds to the vertex of the large hexagon called the large vector (LV), that on the middle side point of the large hexagon called the medium vector (MV), or that on the vertex of the small (inside) hexagon called the small vector (SV), respectively.

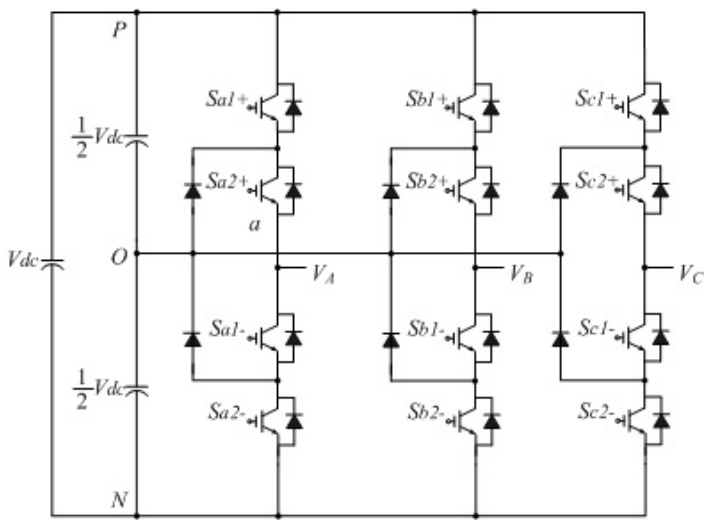

Fig. 1. Three level NPC inverter.

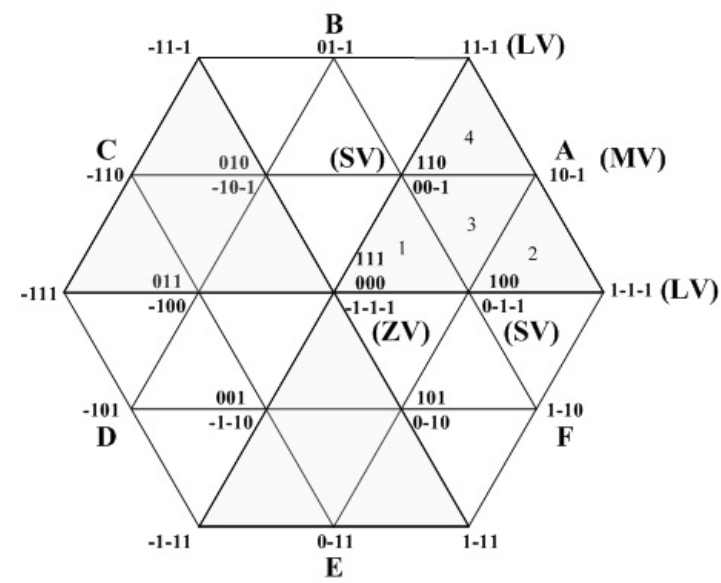

Fig. 2. Hexagon showing voltage vectors of 3-level inverter.

\section{B. Determination of region and sector}

The term sector denotes one of the six large triangles noted as $\mathrm{A}, \mathrm{B}, \mathrm{C}, \mathrm{D}, \mathrm{E}$, or $\mathrm{F}$, and the term regions numbered as 1 , 2, 3 or 4 in Fig. 2 are the four small triangles in a sector. In this paper, for deciding the switching time, the switching times for the reference voltage vector is determined first by using two LVs and one $\mathrm{ZV}$ with the same scheme used in a 2-level SVPWM.

The switching times of the $\mathrm{LVs}$ and the $\mathrm{ZV}$ for $V^{*}$, shown in Fig. 3, are determined by Eqs. (1) and (2), where $d_{m 1}\left(T_{r} / T_{s}\right)$ represents the duty ratio of the right vector of the large triangle, $d_{m 2}\left(T_{l} / T_{s}\right)$ is that of the left vector, $T_{s}$ is the sampling time, and $\gamma$ is the angle between the right vector and the voltage reference.

$$
\begin{gathered}
d_{m 1}=\frac{T_{r}}{T_{s}}=\sqrt{3} \frac{\left|V_{r e f}\right|}{V_{d c}} \sin \left(60^{0}-\gamma\right) \\
d_{m 2}=\frac{T_{l}}{T_{s}}=\sqrt{3} \frac{\left|V_{r e f}\right|}{V_{d c}} \sin (\gamma) .
\end{gathered}
$$

$d_{m 1}$ and $d_{m 2}$ are calculated in the beginning so that these values can be used in further steps for determining the switching times (equivalently duty ratios) in the linear as well as over modulation regions without using trigonometric functions. The region where the reference voltage $V^{*}$ exists can be determined 


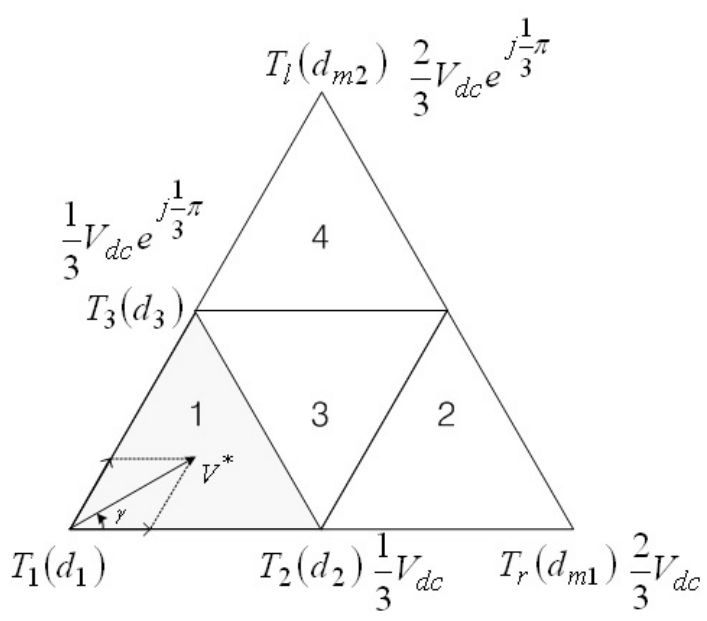

Fig. 3. Reference voltage in region 1 with showing the voltage vectors and corresponding duty ratios.

by the following conditions [11]:

Region 1: $\left(d_{m 1}+d_{m 2}\right) \leq 0.5$
Region 2: $d_{m 1}>0.5$
Region 4: $d_{m 2}>0.5$
Region 3: otherwise

\section{Switching time with respect to each region}

The reference voltage $V^{*}$ in region 1 is generated by applying three voltage vectors consisting of vertices with their switching times determined by the voltage-second rule as in Eq. (4). Eq. (4) is equivalent to Eq. (5) by assuming that the rotating $V^{*}$ is considered as a stationary vector with a constant magnitude during the sampling time $T_{s}$. By substituting the vectors in Eq. (5) with the actual values of the voltage vectors, Eq. (6) can be given. Instead of using switching times ( $T_{1}$ etc.), duty ratios $\left(d_{1}\right.$ etc.) are used in this paper for the convenience of implementation. By dividing Eq. (6) into real and imaginary parts, Eq. (7) can be derived. Where, $d_{1}, d_{2}$, and $d_{3}$ represent the duty ratio of the left-side vector: $V(l)$, the right-side vector: $V(2)$, and the center vector: $V(3)$ in each region, respectively.

$$
\begin{gathered}
\int_{0}^{T_{s}} V^{*} d t=\int_{0}^{T_{1}} V(1) d t+\int_{T_{1}}^{T_{1}+T_{2}} V(2) d t+\int_{T_{2}}^{T_{s}} V(3) d t \\
V^{*} \cdot T_{s}=V(1) \cdot T_{1}+V(2) \cdot T_{2}+V(3) \cdot T_{3} \\
\left|V^{*}\right| e^{j \gamma}=0 \cdot d_{1}+\frac{1}{3} V_{d c} \cdot d_{2}+\frac{1}{3} V_{d c} e^{j \frac{1}{3} \pi} \cdot d_{3} .
\end{gathered}
$$

By comparing Eqs. (1) and (2) with Eq. (7), $d_{1}, d_{2}$, and $d_{3}$ can be expressed as $d_{m 1}$ and $d_{m 2}$, as shown in Eq. (8) [12].

$$
\begin{gathered}
\left|V^{*}\right| \cos \gamma=\frac{1}{3} V_{d c} \cdot d_{2}+\frac{1}{3} V_{d c} \cdot \frac{1}{2} \cdot d_{3} \\
\left|V^{*}\right| \sin \gamma=\frac{1}{3} V_{d c} \cdot \frac{\sqrt{3}}{2} \cdot d_{3} \\
d_{3}=\left|V^{*}\right| \sin \gamma \times \frac{2 \sqrt{3}}{V_{d c}}=2 \times d_{m 2} \\
d_{2}=\frac{3}{V_{d c}} \times\left(\left|V^{*}\right| \cos \gamma-\frac{1}{\sqrt{3}} \cdot\left|V^{*}\right| \sin \gamma\right)=2 \times d_{m 1} .
\end{gathered}
$$

TABLE I

$d_{1}, d_{2}$, AND $d_{3}$ EXPRESSED BY $d_{m 1}$ AND $d_{m 2}$ IN EACH REGION

\begin{tabular}{|c|c|c|c|c|}
\hline Duty Region & 1 & 2 & 3 & 4 \\
\hline$d_{2}$ & $2 d_{m 1}$ & $2 d_{m 1}-1$ & $2 d_{m 1}+2 d_{m 2}-1$ & $2 d_{m 1}$ \\
\hline$d_{3}$ & $2 d_{m 2}$ & $2 d_{m 2}$ & $1-2 d_{m 2}$ & $2 d_{m 2}-1$ \\
\hline$d_{1}$ & \multicolumn{4}{|c|}{$1-d_{2}-d_{3}$} \\
\hline
\end{tabular}

TABLE II

Duty Ratios For Each Region in SECtor A

\begin{tabular}{|c|c|c|}
\hline & Region 1 & Region 2 \\
\hline$S_{a 1}+$ & $d_{1} / 3+d_{2} / 2+d_{3} / 2$ & $d_{1} / 2+d_{2}+d_{3}$ \\
$S_{a 2}+$ & $2 d_{1} / 3+d_{2}+d_{3}$ & $d_{1}+d_{2}+d_{3}$ \\
$S_{b_{1}}+$ & $d_{1} / 3+d_{3} / 2$ & 0 \\
$S_{b 2}+$ & $2 d_{1} / 3+d_{2} / 2+d_{3}$ & $d_{1} / 2+d_{3}$ \\
$S_{c 1}+$ & $d_{1} / 3$ & 0 \\
$S_{c 2}+$ & $2 d_{1} / 3+d_{2} / 2+d_{3} / 2$ & $d_{1} / 2$ \\
\hline & Region 3 & Region 4 \\
\hline$S_{a 1}+$ & $d_{1} / 2+d_{2}+d_{3} / 2$ & $d_{1} / 2+d_{2}+d_{3}$ \\
$S_{a 2}+$ & $d_{1} / 3+d_{2}+d_{3}$ & $d_{1}+d_{2}+d_{3}$ \\
$S_{b 1}+$ & $d_{1} / 2$ & $d_{1} / 2+d_{3}$ \\
$S_{b 2}+$ & $d_{1}+d_{2}+d_{3} / 2$ & $d_{1}+d_{2}+d_{3}$ \\
$S_{c 1}+$ & 0 & 0 \\
$S_{c 2}+$ & $d_{1} / 2+d_{3} / 2$ & $d_{1} / 2$ \\
\hline
\end{tabular}

By applying the same process for different regions, the duty ratios written by $d_{m 1}$ and $d_{m 2}$ in each region can be summarized as Table I. Table I illustrates that the duty ratios are determined with simple algebraic math operations.

\section{Determination of switching times for each switch}

After obtaining the duty ratios of the switching time, it is necessary to determine that for each of the switches such as $S_{a 1}+$. Fig. 4 depicts the switch statuses of $S_{a 1}+$ and $S_{a 2}+$ corresponding to $T_{1}, T_{2}$, and $T_{3}$ (equally $d_{1}, d_{2}$, and $d_{3}$ ) in region 1 . The demanded states for $S_{a 1}+$ and $S_{a 2}+$ are illustrated in the bottom figure of Fig. 4 (b) for generating pole voltage waveforms like $V_{A O}$, shown at the top of the figure.

To generate $V^{*}$ with a minimum switching transition, the voltage vectors are selected with a counter clock wise (CCW) direction from (-1-1-1) vector to (111) and then returned to (-1-1-1) with a clockwise (CW) direction as shown in Fig. 4(a). In other words, (-1-1-1) $\rightarrow$ (0-1-1) $\rightarrow$ (00-1) $\rightarrow$ (000) $\rightarrow(100) \rightarrow(110) \rightarrow(111)$ in $\mathrm{CCW}$ and $(111) \rightarrow . . \rightarrow(000)$ in $\mathrm{CW}$ like the pole voltage waveforms given in Fig. 4. By drawing pictures like the one in Fig. 4, the switching time for each switch can be easily determined. For instance, from Fig 4, it can be determined that $S_{a 1}+$ is gated on during $T_{1} / 3+T_{2} / 2+T_{3} / 2$ (equivalently $d_{1} / 3+d_{2} / 2+d_{3} / 2$ ), and that $S_{a 2}+$ remains on during $2 T_{1} / 3+T_{2}+T_{3}$ (equivalently $2 d_{1} / 3+d_{2}+d_{3}$ ). The duty ratios in sector $\mathrm{A}$ corresponding to each region are summarized in Table II.

By applying the same procedure to the other sectors from B to $\mathrm{F}$, the switching time (duty ratio) can be decided with less effort. Table II shows the switching times for only the upper switches such as $S_{a 1}+$ and $S_{a 2}+$. Since the upper switches $S_{a 1}+$ or $S_{a 1}+$ are acting complementary with the lower switches $S_{a 1}$ or $S_{a 2^{-}}$, the command of the switching times of the bottom two switches such as $S_{a 1}$ - and $S_{a 2}$ - are determined by those of the upper two switches with the subtraction of the calculated duty ratios from $100 \%$ of the duty cycle, i.e. $1-d$. 


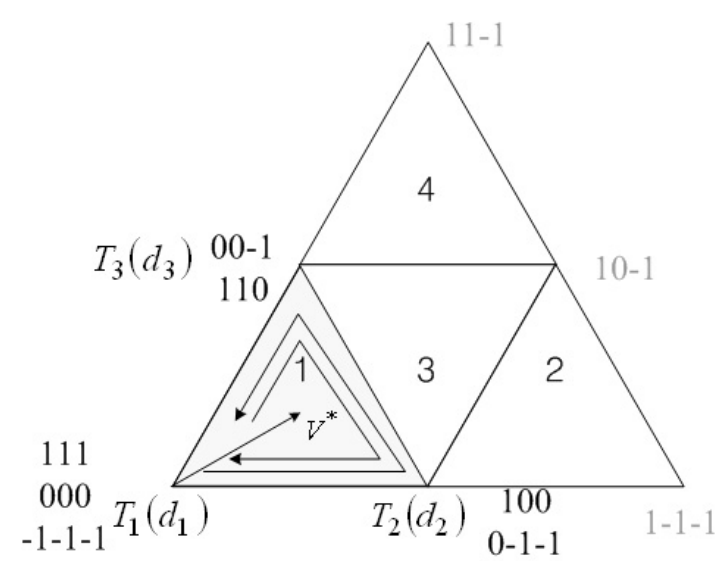

(a)

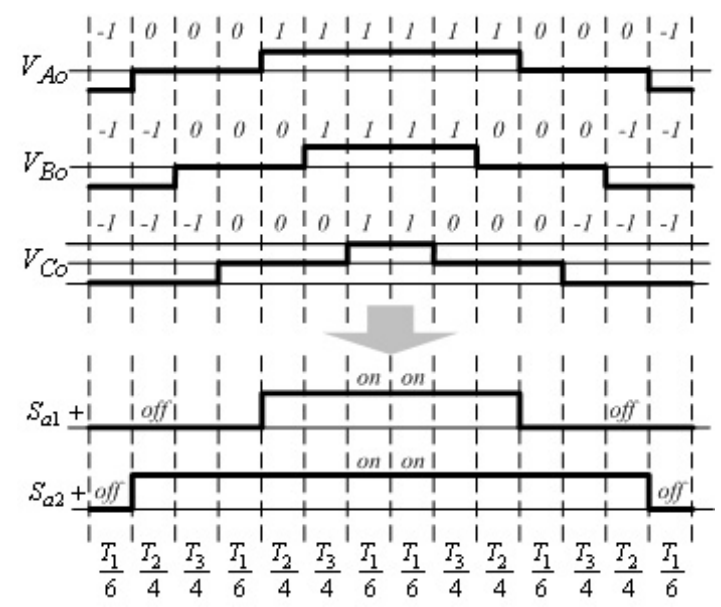

(b)

Fig. 4. (a) Sequence of selecting voltage vectors. (b) Inverter pole voltage waveforms and related gate signals.

\section{SVPWM IN OVERMODULATION REGION}

In the SOM, the magnitude and the phase of the reference voltage are modified to increase the magnitude of the output voltage and to have the linearity of the inverter output voltage with respect to the modulation index $m$ [9], [10]. As in 2-level inverters, the overmodulation occurs when $V^{*}$ for the 3-level inverter is located outside the large hexagon as shown in Fig. 5(b). Fig. 5 shows that the side of the large hexagon of the 3-level SVPWM can be considered to be the same as the one for the 2-level SVPWM. One sector for the three-level case is matched with one of the small triangles in the hexagon for the 2-level SVPWM.

The proposed SVPWM method is devised to use $d_{m 1}$ and $d_{m 2}$, which are equivalent to $T_{r}$ and $T_{l}$ in the 2-level SVPWM, for realizing the SVPWM scheme for a 3-level inverter with the features of simplicity and easy implementation. In addition, the proposed method avoids using the time consuming calculation processes, such as trigonometric functions in the determination of the reference voltage vector and the switching time itself, used in conventional methods.

\section{A. SOM in mode I $(0.9069 \leq m<0.9514)$}

The modulation index $m$ is defined as Eq. (9). It is the ratio of the fundamental component of the voltage reference to that

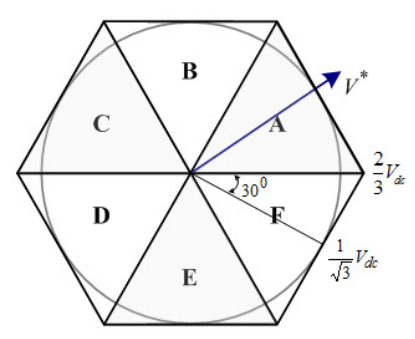

(a)

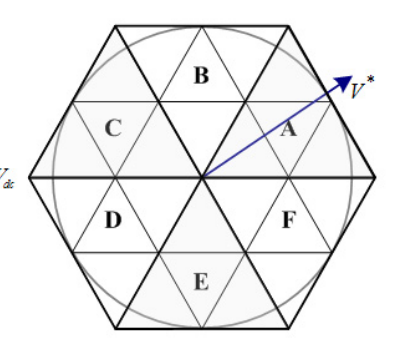

(b)
Fig. 5. Reference voltage vector of (a) 2-level. (b) 3-level systems in the overmodulation region.

for the six-step operation.

$$
m=V_{1}^{*} / V_{1, \text { Six-step }}=V_{1}^{*} / \frac{2}{\pi} V_{d c} .
$$

According to the $m$ value, the SOM scheme needs different strategies for generating a reference voltage vector. The range of the overmoduation is divided by two, and called mode I or mode II. In mode I, the magnitude of the voltage reference is modified to have a boosted value. On the other hand, both the magnitude and the phase angle of the reference voltage need to be modified in mode II [9], [10]. In mode I, the magnitude of the new voltage reference is boosted in order to have the same fundamental voltage level corresponding to $m$. In this paper, the amount of boosting is determined by FSE, and the process is explained below.

The voltage reference $V^{*}$ can be expressed as Eq. (10). Where, $V_{1}$ is the magnitude of original reference voltage. Because $V_{1}$ is larger than $V_{d c} / \sqrt{3}$, during the rotation of the reference vector, the vector goes outside the hexagon during the period $1 \sim 2$ as depicted in Fig. 6 (a). During the period $1 \sim 2$, the voltage magnitude is limited to $V_{d c} / \sqrt{3} \cos (\pi / 6-\theta)$, and the averaged voltage magnitude becomes less than $V_{1}$. Therefore, a modification of the voltage references is required as shown in Eq. (11) and Fig. 6 (b), where $V_{2}$ represents the boosted voltage magnitude to compensate the decrease in the voltage magnitude due to the overmodulation in $1 \sim 2$ period. Where $\alpha_{c}$ is the crossover angle.

$$
\begin{gathered}
V^{*}=V_{1} e^{j \theta} \quad 0 \leq \theta \leq \frac{\pi}{3} \\
V_{\text {new }}^{*}=\left\{\begin{array}{cc}
V_{2} e^{j \theta} & 0 \leq \theta<\alpha_{c} \\
\left.V_{d c} / \sqrt{3} \cos (\pi / 6-\theta)\right)^{j \theta} & \alpha \leq \theta<\frac{\pi}{3}-\alpha_{c} \\
V_{2} e^{j \theta} & \frac{\pi}{3}-\alpha_{c} \leq \theta<\frac{\pi}{3}
\end{array}\right.
\end{gathered}
$$

Fig. 6(b) illustrates that during the periods A-B and C$\mathrm{D}$, the reference vector having a $V_{2}$ magnitude is located inside the hexagon, and during the C-D periods the reference voltage moves along the side of the large triangle, and whose magnitude is expressed as $V_{d c} / \sqrt{3} \cos (\pi / 6-\theta)$ in Eq. (11). If the modified voltage reference $\left(V^{*}{ }_{n e w}\right)$ is selected by Eq. (11), it is necessary to calculate trigonometric functions such as $\cos (\pi / 6-\theta)$ with the information of $\alpha_{c}$ and the angle of the reference vector as was done in [4] and [5].

To remove this complexity, the proposed SOM generates a new boosted voltage vector with a simple procedure, where the $V_{2}$ value is directly obtained from the modulation index $m$ without finding $\alpha_{c}$ and the angle of the reference vector. 


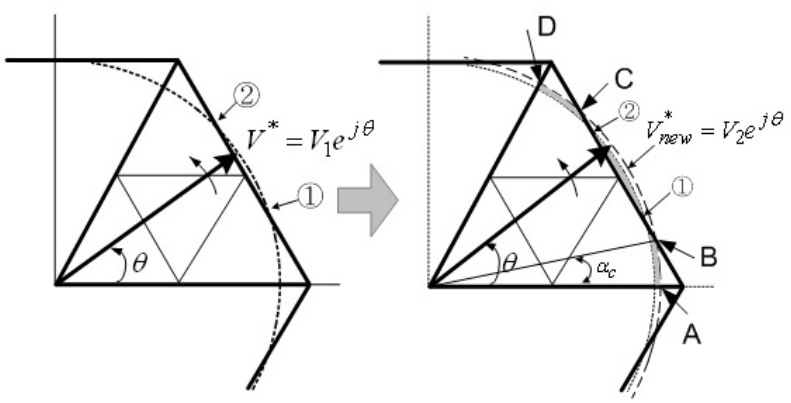

Fig. 6. (a) Original vector before boosting. (b) New reference voltage vector after boosting in mode I with showing $\alpha_{c}$.

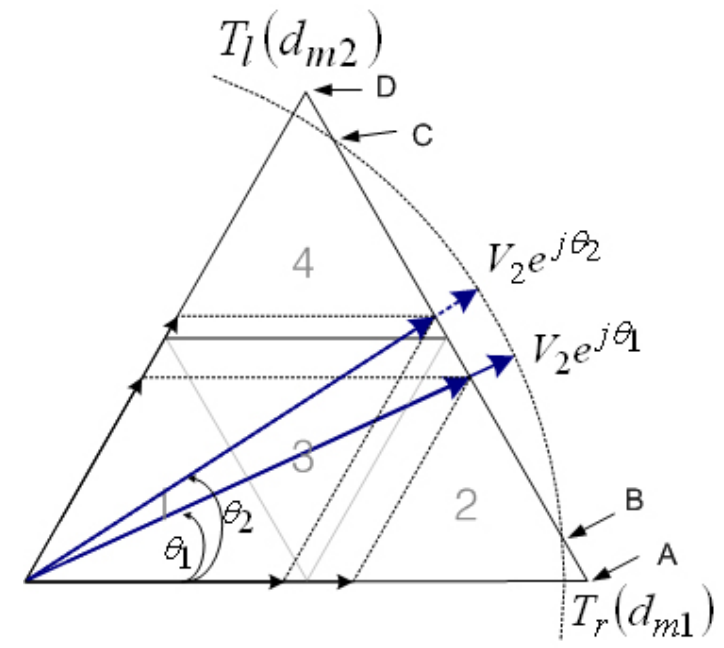

Fig. 7. New reference voltage generation by minimum phase error projection scheme.

The duty ratios are selected by utilizing the minimum phase error projection scheme shown in Fig. 7 and expressed as Eq. (12), when $V^{*}{ }_{n e w}$ having a $V_{2}$ magnitude is located outside the hexagon such as during the periods B-C like $V_{2} e^{j \theta_{1}}$, and $V_{2} e^{j \theta_{2}}$. The $V^{*}{ }_{n e w}$ vector is projected on the side of the hexagon. As the magnitude of the projected vector in the periods B-C (equivalently $\alpha_{c} \leq \theta<\pi / 3-\alpha_{c}$ ) is identical to $V_{d c} / \sqrt{3} \cos (\pi / 6-\theta)$, Eq. (11-b) is automatically satisfied. Consequently, the duty ratios for $V_{\text {new }}^{*}$ expressed in Eq. (13) is determined by Eq. (12) for the case where $d_{m 1}+d_{m 2}>1$ occurred in the periods B-C.

$$
\begin{gathered}
d_{m 1}^{\text {new }}=\frac{d_{m 1}}{d_{m 1}+d_{m 2}}, \quad d_{m 2}^{\text {new }}=\frac{d_{m 2}}{d_{m 1}+d_{m 2}} \\
V_{\text {new }}^{*}=V_{2} e^{j \theta} \quad 0 \leq \theta \leq \frac{\pi}{3} .
\end{gathered}
$$

In Eq. (12), $d_{m 1}$ and $d_{m 2}$ are the duty ratios for $V^{*}$ new generated by the large vectors, and $d_{m 1}^{\text {new }}$ and $d_{m 2}^{\text {new }}$ are the recalculated duty ratios obtained from the projected $V^{*}$ new.

The required voltage level, i.e. $V_{2}$, which makes the fundamental voltage magnitude after boosting equivalent to $V_{1}$, is calculated by employing the FSE. By equalizing the coefficient of the FSE of the original vector with that of the new vectors shown as Eq. (14), $V_{2}$ can be determined. For simplicity, the constant coefficients of the FSE on both sides of Eq. (14) are omitted.

$$
\begin{aligned}
& \int_{0}^{\frac{\pi}{3}} V_{1} e^{j \theta} e^{-j \theta} d \theta= \\
& \int_{0}^{\alpha_{c}} V_{2} e^{j \theta} e^{-j \theta} d \theta+\int_{\alpha_{c}}^{\pi / 3^{-\alpha_{c}}} \frac{V_{d c}}{\sqrt{3} \cos \left(\pi / 6^{-\alpha_{c}}\right)} e^{j \theta} e^{-j \theta} d \theta \\
& +\int_{\pi / 3}^{\pi / 3} V_{3} V_{c} e^{j \theta} e^{-j \theta} d \theta
\end{aligned}
$$

By using the relation $\int \frac{1}{\cos \xi} d \xi=\ln \tan \left(\frac{\pi}{4}+\frac{\xi}{2}\right)$, Eq. (14) can be written as:

$$
V_{1}=\frac{6}{\pi} \alpha_{c} V_{2}+\frac{\sqrt{3}}{\pi} V_{d c}\left[\ln \tan \left(\frac{\pi}{3}-\frac{\alpha_{c}}{2}\right)-\ln \tan \left(\frac{\pi}{6}-\frac{\alpha_{c}}{2}\right)\right]
$$

Fig. 6(b) clearly shows that the magnitude $V_{2}$ is the value at which $\theta=\alpha_{c}$, so that the following equation is obtained:

$$
V_{2}=\frac{V_{d c}}{\sqrt{3}} \frac{1}{\cos \left(\frac{\pi}{6}-\alpha_{c}\right)}
$$

Substituting Eq. (16) into (15), the relationship between $V_{1}$ and $\alpha_{c}$ can be given as Eq. (17):

$$
\begin{aligned}
& V_{1}=\frac{6}{\pi} \times \alpha_{c} \times \frac{V_{d c}}{\sqrt{3}} \times \frac{1}{\cos \left(\frac{\pi}{6}-\alpha_{c}\right)}+ \\
& \frac{\sqrt{3}}{\pi} \times V_{d c}\left[\ln \tan \left(\frac{\pi}{3}-\frac{\alpha_{c}}{2}\right)-\ln \tan \left(\frac{\pi}{6}-\frac{\alpha_{c}}{2}\right)\right]
\end{aligned}
$$

With Eqs. (15)-(17), $V_{2}$ corresponding to $V_{1}$ can be decided and shown as Fig. 8. $V_{1}$, and $V_{2}$ in above equations are equivalent to $m_{v 1}$, and $m_{v 2}$, respectively. The definitions of $m_{v 1}$ and $m_{v 2}$ are given as:.

$$
m=m_{v 1}=\frac{V_{1}^{*}}{2 / \pi^{V_{d c}}}, m_{v 2}=\frac{V_{i n s t}^{*}}{2 / \pi^{V_{d c}}} .
$$

$V_{1}{ }^{*}$ is the fundamental component of the voltage reference. On the other hand, $V_{\text {inst }}{ }^{*}$ in $m_{v 2}$ is the instantaneous value of the reference voltage. In implementation, the magnitude of the modified reference is not a fundamental but an instantaneous value. Therefore, in the proposed SOM, $m_{v 2}$ defined with an instantaneous value is used. Where, the modulation index $m$ with respect to $\alpha_{c}$ can be given as Eq. (19) [13]. Hence, it can be seen that mode I starts at $m=0.907$ and ends at $m=$ 0.9514 for $\alpha_{c}=30^{0}$ and $0^{0}$, respectively.

$$
m=\frac{\sqrt{3} \ln \left[\tan \left(\frac{\pi}{3}-\frac{\alpha_{c}}{2}\right)\right]}{1-\frac{6}{\pi} \alpha_{c}} .
$$

Mode I ends at $\alpha_{c}=0^{0}$. From Eq. (16) it can be seen that $V^{*}{ }_{i n s t}$ is $2 / 3 V_{d c}$, and that it results in $m_{v 2}=1.0472$ as Eq. (21).

$$
\begin{aligned}
& V_{2}=\frac{V_{d c}}{\sqrt{3}} \frac{1}{\cos \left(\frac{\pi}{6}\right)}=\frac{2}{3} V_{d c} \\
& m_{v 2}=V_{2} / \frac{2}{\pi} V_{d c}=\frac{2}{3} V_{d c} / \frac{2}{\pi} V_{d c}=\frac{\pi}{3}=1.0472 .
\end{aligned}
$$

The result shows that $m_{v 1}$ for $V_{1}^{*}$ varies from $m=0.907$ to 0.9514 in mode I. On the other hand $m_{v 2}$ varies from 0.907 to 1.0472. This agrees with the $\mathrm{x}$ and $\mathrm{y}$ axis values in Fig. 8 . In implementation, $m_{v 2}$ with respected to $m_{v 1}$ is determined by the approximate equations summarized in Table III. It is divided in 3 sections and obtained with the help of a curve fitting tool. 


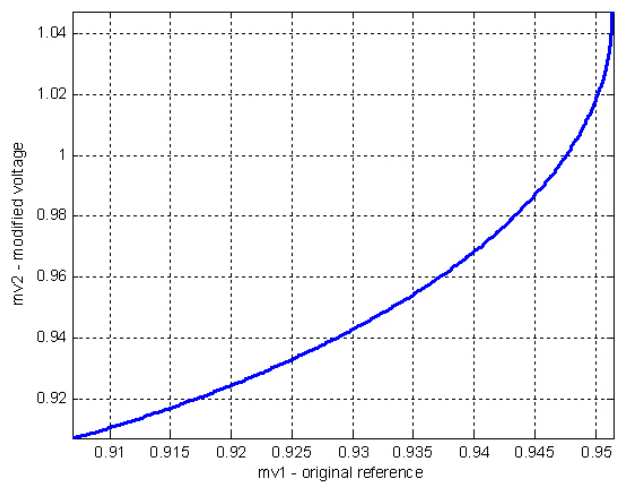

Fig. 8. Relationship between $m_{v 2}$ and $m_{v 1}$ in mode I.

TABLE III

ApPRoXimate EQUATION FOR $m_{v 2}$ IN MODE IWITH RESPECT TO $m_{v 1}$ DIVIDED IN THREE SECTIONS

\begin{tabular}{|l|l|}
\hline \multicolumn{2}{|c|}{ mode I $(0.9069 \leq m<0.9514)$} \\
\hline$m_{v 1}($ original $m)$ & $m_{v 2}($ modified $m)$ \\
\hline $0.9069 \leq m_{v 1}<0.940$ & $m_{v 2}=1.731 m_{v 1}-0.6656$ \\
\hline $0.940 \leq m_{v 1}<0.951$ & $m_{v 2}=5.48 m_{v 1}-4.19$ \\
\hline $0.951 \leq m_{v 1}<0.9514$ & $m_{v 2}=35.82 m_{v 1}-33.04$ \\
\hline
\end{tabular}

It should be mentioned that in mode I, the only required value is $m_{v 2}$ equivalently $V_{2}$ (or $V^{*}{ }_{i n s t}$ ). This value is determined by Table III, and the duty ratios for $V^{*}{ }_{n e w}$ having a $V_{2}$ magnitude is automatically decided by Eq. (12) when $V^{*}$ new goes outside the hexagon.

\section{B. SOM in mode II $(0.9514 \leq m \leq 1)$}

In mode II, the fundamental component of $V_{1}$ cannot be generated by only boosting the voltage magnitude [9], [10]. In the conventional method for the overmodulation of 2-level inverters, the voltage and the angle reference are generated by Eqs. (22) and (23) [13], where $\alpha_{h}$ represents the holding angle. Fig. 9 illustrates the SOM operation for 3-level inverters in mode II. Fig. 9 shows that when the angle of the reference vector $(\theta)$ is located between 0 and $\alpha_{h}$, the new reference vector remains at 0 degrees. Between $\alpha_{h}$ and $\pi / 3-\alpha_{h}$ it goes along the side of the hexagon, and it remains at $\pi / 3$ when $\theta$ is located between $\pi / 3-\alpha_{h}$ to $\pi / 3$.

$$
\begin{gathered}
V^{*}=\left\{\begin{array}{cc}
\frac{2}{3} V_{d c} & \leq \theta<\alpha_{h} \\
\frac{V_{d c}}{\sqrt{3} \cos \left(\frac{\pi}{6}-\theta\right)} e^{j \theta} & \alpha_{h} \leq \theta<\frac{\pi}{3}-\alpha_{h} \\
\frac{2}{3} V_{d c} e^{j \frac{\pi}{3}} & \frac{\pi}{3}-\alpha_{h} \leq \theta<\frac{\pi}{3}
\end{array}\right. \\
\theta^{*}=\left\{\begin{array}{cc}
0 & 0 \leq \theta<\alpha_{h} \\
\frac{\theta-\alpha_{h}}{\frac{\pi}{6}-\alpha_{h}} \cdot \frac{\pi}{6} & \alpha_{h} \leq \theta<\frac{\pi}{3}-\alpha_{h} . \\
\frac{\pi}{3} & \frac{\pi}{3}-\alpha_{h} \leq \theta<\frac{\pi}{3}
\end{array}\right.
\end{gathered}
$$

In the proposed method for 3-level inverters, the voltage and the angle reference are given as Eqs. (24) and (25). The magnitude of $V^{*}$ new is fixed as $2 / 3 V_{d c}$, which is the

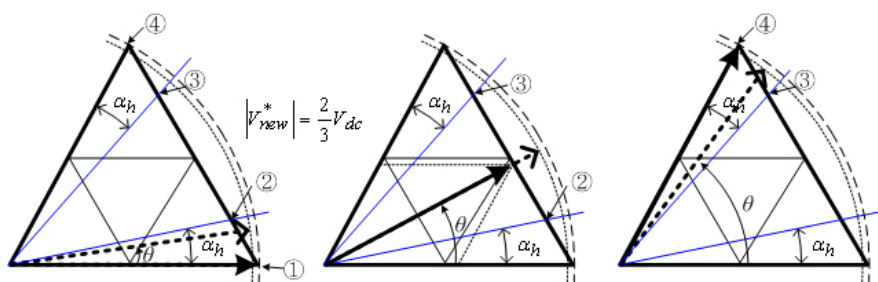

Fig. 9. $V^{*}$ in mode II discriminated in 3 sections by the phase reference with respect to $\alpha_{h}$

maximum inverter voltage equivalent to $m_{v 2}=1.0472$. The angle reference is given by Eq. (25), to make the SVPWM method simple.

$$
V_{\text {new }}^{*}=\frac{2}{3} V_{d c} e^{j \theta} 0 \leq \theta<\frac{\pi}{3} .
$$

In mode II, it can be seen that the reference vector described in Eq. (22) for the conventional method of 2-level SVPWM always moves along the side of the hexagon or stays in the vertices of the triangle.

$$
\theta^{*}=\left\{\begin{array}{cc}
0 & 0 \leq \theta<\alpha_{h} \\
\theta & \alpha_{h} \leq \theta<\frac{\pi}{3}-\alpha_{h} \\
\frac{\pi}{3} & \frac{\pi}{3}-\alpha_{h} \leq \theta<\frac{\pi}{3}
\end{array} .\right.
$$

Therefore, $V^{*}{ }_{\text {new }}$ in Eq. (24), having a fixed magnitude of $2 / 3 V_{d c}$, always rotates outside the large hexagon. With the angle command of Eq. (25) it can generate the same voltage reference as that shown in Eq. (22). Also, as in mode I, the duty ratios for generating $V^{*}$ new can be easily determined by the identical projection scheme described in Eq. (12).

Since, in the proposed SOM for mode II, $m_{v 2}$ is fixed at 1.0472 (equivalently $2 / 3 V_{d c}$ ), only expressing $\alpha_{h}$ as a function of $m_{v 1}$ is necessary. The relationship between $\alpha_{h}$ and $m_{v 1}$ is determined in a method that is similar to the one for obtaining $m_{v 2}$ in mode I. In other words, by using the FSE, $\alpha_{h}$ with respect to $m_{v 1}$ is found. By equalizing the coefficient of the FSE of the original vector expressed as Eq. (26) with that of the new vector, Eqs. (27) and (28) can be obtained.

$$
\begin{gathered}
V^{*}=V_{1} e^{j \theta} \quad 0 \leq \theta \leq \frac{\pi}{3} \\
\int_{0}^{\frac{\pi}{3}} V_{1} d \theta=\int_{0}^{\alpha_{h}} \frac{2}{3} V_{d c} e^{-j \theta} d \theta+\int_{\alpha_{h}}^{\frac{\pi}{3}-\alpha_{h}} \frac{V_{d c}}{\sqrt{3} \cos \left(\frac{\pi}{6}-\theta\right)} d \theta+ \\
\int_{\frac{\pi}{3}-\alpha_{h}}^{\frac{\pi}{3}} \frac{2}{3} V_{d c} e^{j\left(\frac{\pi}{3}-\theta\right)} d \theta \\
V_{1} \cdot \frac{\pi}{3}=\frac{2}{3} V_{d c} \cdot j\left[e^{-j \alpha_{h}}-1\right]+ \\
\frac{V_{d c}}{\sqrt{3}} \cdot \int_{\alpha_{h}}^{\frac{\pi}{3}-\alpha_{h}} \frac{V_{d c}}{\cos \left(\frac{\pi}{6}-\theta\right)} d \theta+\frac{2}{3} V_{d c} \cdot j\left[1-e^{-j \alpha_{h}}\right] .
\end{gathered}
$$

Eq. (29) is given by dividing Eq. (28) into the imaginary and real parts.

$$
\begin{aligned}
& V_{1}=\frac{4}{\pi} \cdot V_{d c} \cdot 2 \cdot \sin \alpha_{h}+ \\
& \quad \frac{\sqrt{3}}{\pi}\left[\ln \tan \left(\frac{\pi}{3}-\frac{\alpha_{h}}{2}\right)-\ln \tan \left(\frac{\pi}{6}-\frac{\alpha_{h}}{6}\right)\right] .
\end{aligned}
$$




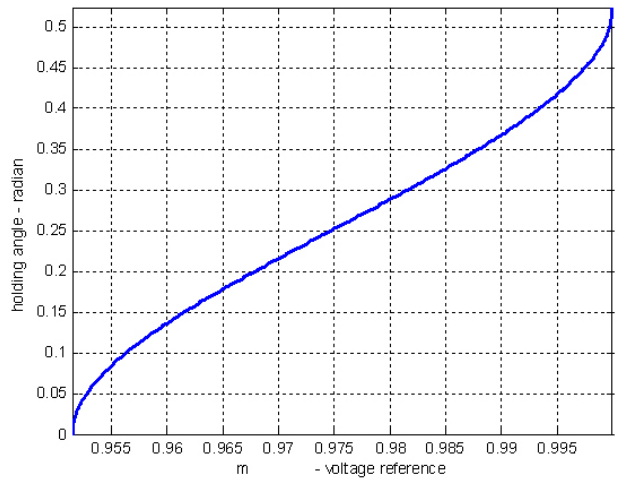

Fig. 10. Holding angle $\alpha_{h}$ with respect tom in mode II.

TABLE IV

Approximate Equations for Holding Angle in Mode II Corresponding to $\mathrm{M}_{v 1}$ Divided in ThreE SECTIONS

\begin{tabular}{|c|c|}
\hline \multicolumn{2}{|c|}{ mode II $\left(0.9514 \leq m_{v 1} \leq 1\right)$} \\
\hline$m_{v 1}(m)$ & $\alpha_{h}$, holding angle \\
\hline $0.9514 \leq m_{v 1}<0.955$ & $\alpha_{h}=20.73 m_{v 1}-19.71$ \\
\hline $0.955 \leq m_{v 1}<0.995$ & $\alpha_{h}=7.797 m_{v 1}-7.351$ \\
\hline $0.995 \leq m_{v 1}<1$ & $\alpha_{h}=19.87 m_{v 1}-19.37$ \\
\hline$m_{v 1}=1$ & $\alpha_{h}=\pi / 6$ \\
\hline
\end{tabular}

By substituting $V_{1}$ with $m=m_{v 1}=V_{1} /\left(2 V_{d c} / \pi\right)$, the relationship between $m$ (or $m_{v 1}$ ) and $\alpha_{h}$ can be given as Eq. (30), and drawn as Fig. 10.

$$
m=2 \cdot \sin \alpha_{h}+\frac{\sqrt{3}}{2}\left[\ln \tan \left(\frac{\pi}{3}-\frac{\alpha_{h}}{2}\right)-\ln \tan \left(\frac{\pi}{6}-\frac{\alpha_{h}}{6}\right)\right] .
$$

Similar to mode I, with the help of a curve fitting tool the approximate equations for the holding angle $\alpha_{h}$ as a function of the modulation index $m$ can be found and summarized in Table IV.

In summary, only $\alpha_{h}$ corresponding to $m\left(\mathrm{~m}_{v 1}\right)$ is determined in mode II, and the duty ratio for $V^{*}{ }_{n e w}$ is determined by a projection scheme identical to the one described in Eq. (12). Fig. 11 shows a flowchart of the proposed over modulation method for 3-level inverters.

\section{VERIFICATIONS}

The specifications are summarized in Table V. In order to verify the validity of the proposed SOM, the algorithm has been implemented by using a digital signal processor (TMS320F28335) with the V/F control of an induction motor. The switching frequency is chosen as $2 \mathrm{KHz}$ with the consideration of high power converters for high speed trains, and motors are under controlled from the linear to six-step operation mode. In the PWM interrupt service routine, the new reference of magnitude of $V^{*}{ }_{n e w}$ (equivalently $m_{v 2}$ ) and the phase angle are generated by the proposed algorithm, shown in Fig. 11. The computation times of the conventional and the proposed methods have been measured and summarized in Table VI. The time for determining the duty ratios has been compared. As expected, Table VI shows that the proposed method has greatly reduced the computation time for the operation of overmodulation mode I as well as mode II.

Fig. 12 shows the experimental results corresponding to various $m$ values. In each figure, the phase current and the

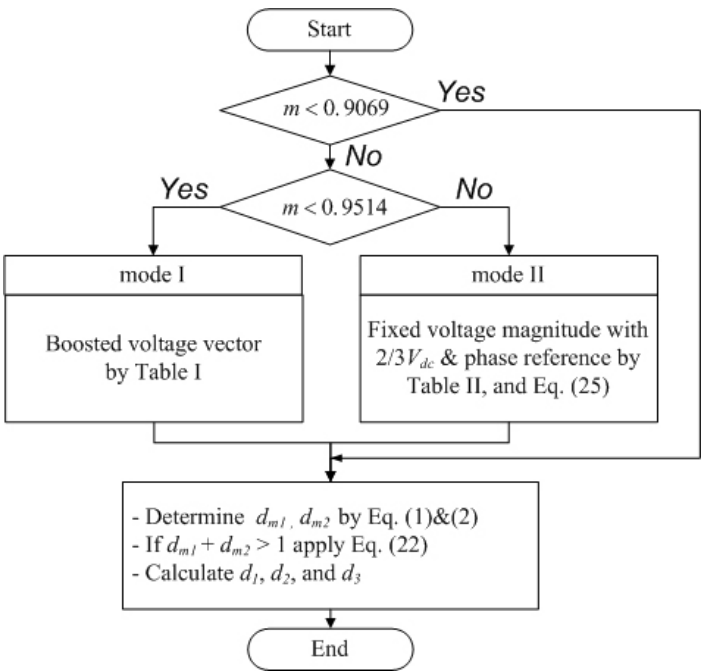

Fig. 11. Flow chart for the proposed SOM.

TABLE V

SPECIFICATIONS FOR EXPERIMENTS

\begin{tabular}{|c|c|}
\hline Motor & $\begin{array}{c}\text { Induction motor (2 } \\
\text { HP/4poles) }\end{array}$ \\
\hline DSP & TMS320F28335 \\
\hline Switching freq. & $2 \mathrm{kHz}$ \\
\hline
\end{tabular}

line voltage waveform of the inverter are shown from top to bottom. Fig. 12(a) shows the experimental results when the motor speed is $500 \mathrm{rpm}$. Since, the voltage vector is rotating inside the small hexagon of Fig. 2, only the small vectors are chosen so that the line voltage looks like that from a 2-level inverter. Fig. 12(b) shows the waveforms for 900rpm. In this operation condition, where the reference voltage is rotating between the small and large hexagon areas, the line voltage shows the conventional waveforms of a 3-level inverter in the linear region. The waveforms obtained for mode I and mode II are shown in Fig. 12(c) and 12(d), respectively. It can be seen that as $m$ increases, the area of missing PWM pulses increases. When $m=1$ (six-step operation), the inverter will generate a square shape waveform, and the waveforms for this operational condition are illustrated in Fig. 12(d).

\section{CONCLUSION}

In this paper, a novel SVPWM including the SOM for 3-level NPC inverters has been proposed. Only the boosted magnitude or the holding angle with respect to the modulation index in mode I or II need to be calculated. The modified voltage reference and the duty ratios have been determined by the minimum phase error projection scheme. As the result, the proposed SOM features a simple calculation process. The relationship between the voltage magnitude and the holding angle corresponding to the modulation index has been determined in advance with the help of the Fourier series expansion and implemented with the approximate equations. Experimental

TABLE VI

COMPARISON OF COMPUTATION TIMES

\begin{tabular}{|c|c|c|}
\hline Operation & Conventional & $\begin{array}{c}\text { Proposed } \\
\text { method }\end{array}$ \\
\hline Linear & $2.95 \mu \mathrm{s}$ & $2.95 \mu \mathrm{s}$ \\
\hline OVM mode I & $10.95 \mu \mathrm{s}$ & $7.04 \mu \mathrm{s}$ \\
\hline OVM mode II & $8.39 \mu \mathrm{s}$ & $6.07 \mu \mathrm{s}$ \\
\hline
\end{tabular}




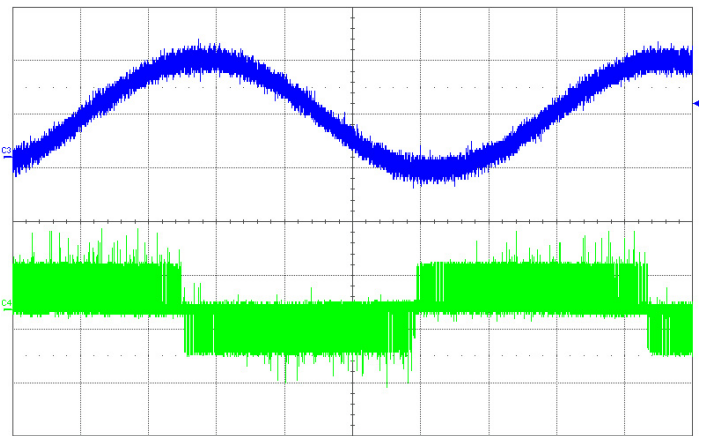

(a)

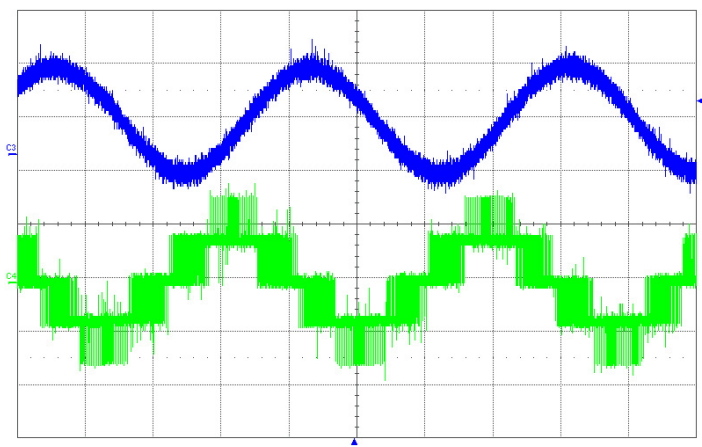

(b)

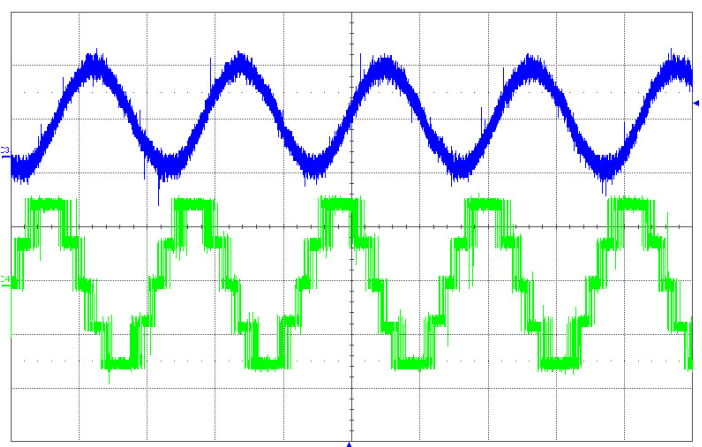

(c)

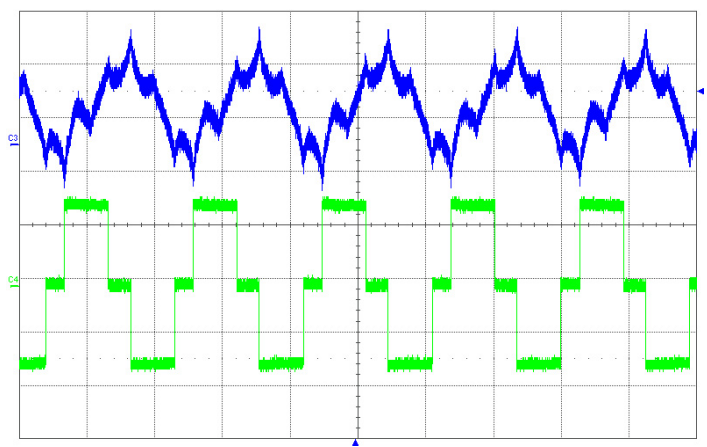

(d)

Fig. 12. Experimental results for (a) motor speed 500rpm: linear region inside the small hexagon, (b) $900 \mathrm{rpm}$ : linear region inside the large hexagon, (c) $1600 \mathrm{rpm}$ : SOM in mode I, (d) $1750 \mathrm{rpm}$ : SOM in mode II especially six-step mode (from top to bottom motor current [1.5A/div.], and line to line inverter output voltage [200V/div.] with time scale [10msec/div.]). results have demonstrated the validity of the proposed SOM for 3-level NPC inverters.

\section{ACKNOWLEDGMENT}

This research was supported by the Basic Science Research Program through the National Research Foundation of Korea (NRF) funded by the Ministry of Education, Science and Technology (No. 2011-0004693).

\section{REFERENCES}

[1] R. Teodorescu, F. Beaabjerg, J.K. Pedersen, E. Cengelci, S.U. Sulistijo, B.O. Woo, and P. Enjeti, "Multilevel converters-A survey," in Proc. EPE Conf., pp. 2-11, 1999.

[2] J. Rodriguez, J. S. Lai, and F. Z. Peng, "Multilevel inverters: a survey of topologies, controls and applications," IEEE Trans. Ind. Electron., Vol. 49, No. 4, pp. 724-738, Aug. 2002.

[3] A. Nabae, I. Takahashi, and H. Akagi, "A new neutral point clamped PWM inverter," IEEE Trans. Ind. Appl., Vol. 17, No. 5, pp. 518-523, Sep. 1981.

[4] S. K. Mondal, B. K. Bose, V. Oleschuk, and J .O. P. Pinto, "Space vector pulse width modulation of three-level inverter extending operation into overmodulation region," IEEE Trans. Power Electron., Vol. 18, No. 2 , pp. 604-611, Mar. 2003.

[5] K. M. Kwon, J. M. Lee, J. M. Lee, and J. H. Choi, "SVPWM overmodulation scheme of three-level inverters for vector controlled induction motor drives," Journal of Power Electron., Vol. 9, No. 3, pp. 481-490, May 2009.

[6] A. K. Gupta, and A. M. Khambadkone, "A simple space vector PWM scheme to operate a three-level NPC inverter at high modulation index including overmodulaton region with neutral point balancing," IEEE Trans. Ind. Appl., Vol. 43, No. 3, pp. 751-760, May/Jun. 2007.

[7] A. Tripathi, A. M. Khambadkone, and S. K. Panda, "Stator flux based space-vector modulation and closed loop control of the stator flux vector in overmodulation into six-step mode," IEEE Trans. Power Electron. Vol. 19, No. 3, pp.775-782, May 2004.

[8] T.G. Habetler, "A space vector-based rectifier regulator for ac/dc/ac converters," in Proc. EPE, Vol. 2, pp. 101-107, 1991.

[9] J. Holtz, W. Lotzkat, and A. M. Khambadkone, "On continuous control of PWM Inverters in the overmodulation range including the six-step mode," IEEE Trans. Power Electron., Vol. 8, No. 4 , pp. 546-553, Oct. 1993.

[10] D. C. Lee, and G. M. Lee, "A novel overmodulation technique for spacevector PWM inverters," IEEE Trans. Power Electron., Vol. 13, No. 6, pp. 1144-1151, Nov. 1998.

[11] A. Kocalmis, and S. Sunter, "Simulation of a space vector PWM controller for a three-level voltage-fed inverter motor drive," IECON 2006, pp. 1915-1920, Nov. 2006.

[12] D. M. Lee, and C. H. Hong "Modeling of SVPWM and control method for driving systems of high-speed trains by using multi-level power converters," Journal of the KIIEE, Vol. 23, No. 12, pp. 136-145, Dec. 2009.

[13] J. H. Bae, Y. C. Kim, C. Y. Won, J. M. Choi, S.W. Ki, and K. H. Bae, "A output voltage linearization in overmodulation region of the space vector PWM," Journal of the Inst. of Electr. Engineers of Korea, Vol. 36, No. 11, pp. 1374-1385, Nov. 1999.

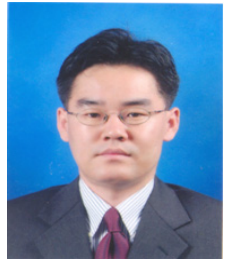

Dong-Myung Lee received his B.S. and M.S. in Electrical Engineering from Hanyang University, Seoul, Korea, in 1994 and 1996, respectively, and his Ph.D. in ECE from the Georgia Institute of Technology, Atlanta, in 2004. From 1996 to 2000, he worked for LG Electronics Inc., Seoul, Korea. From 2004 to 2007, he was employed by the Samsung SDI R\&D Center, Yongin, Korea, as a Senior Engineer. From 2007 to 2008, he was with the Department of Electrical Engineering, Hanyang University, as a Research Professor. Since 2008, he has been an Assistant Professor with the School of Electronic and Electrical Engineering, Hongik University, Seoul, Korea. His research interests include variable speed drives, power quality compensation devices, and power conversion systems for renewable energy sources. 


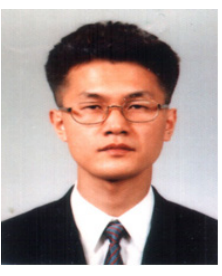

Jin-Woo Jung received his B.S. and M.S. in Electrical Engineering from Hanyang University, Seoul, Korea in 1991 and 1997, respectively, and his Ph.D. in ECE from Ohio State University, Columbus, in 2005. From 1997 to 2000, he was with the Digital Appliance Research Lab., LG Electronics Co., Ltd., Seoul, Korea. From 2005 to 2008, he worked at the R\&D Center, Samsung SDI Co., Korea, as a Senior Engineer. Since 2008, he has been an Assistant Professor with the Division of Electronics and Electrical Engineering, Dongguk University, Seoul, Korea. His current research interests include electric machine drives, the control of distributed generation systems, and the driving circuits and driving methods of ac PDP.

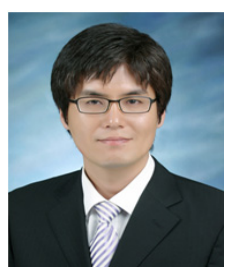

Sang-Shin Kwak received his B.S. and M.S. in Electronics Engineering from Kyungpook National University, Daegu, Korea, in 1997 and 1999, respectively, and his Ph.D. in Electrical Engineering from Texas A\&M University, College Station, in 2005. From 1999 to 2000 , he worked as a Research Engineer at LG Electronics, Changwon, Korea. He was also with the Whirlpool R\&D Center, MI, in 2004. From 2005 to 2007, he worked as a Senior Engineer in Samsung SDI, Yongin, Korea. From 2007 to 2010, he worked as an Assistant Professor at Daegu University, Korea. Since 2010, he has been an Assistant Professor at Chung-Ang University, Seoul, Korea. His research interests include topology design, modeling, control, and analysis of power converters for hybrid/electric vehicle systems, renewable energy systems, adjustable speed drives and digital display drivers. 